\title{
Abdominoplastia en pacientes posbariátricos. Técnicas más usadas
}

\author{
Abdominoplasty in postbariatric patients. Most used techniques
}

\author{
Carlos Matías Luján
}

\section{ÍNDICE}

1. Resumen. .2

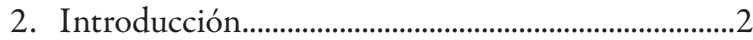

3. Objetivo........................................................................

4. Obesidad y tratamientos quirúrgicos ........................ 3

5. Valoración de pacientes posbariátricos en plan de cirugía de contorno corporal . ...5

6. Premisas para la selección del candidato a la cirugía.

7. Evaluación de distrofias abdominales. . .5

8. Tratamiento de la pared abdominal. ...7

9. Tratamiento de las distrofias abdominales ..............9

a. Paniculectomía prebariátrica 11

b.Abdominoplastia en flor de lis.... 13

c. Abdominoplastia en cinturón (abdominoplastia circunferencial)

d. Levantamiento corporal...................................... 17

e. Cuidado posoperatorio ........................................ 22

f. Complicaciones:................................................. 23

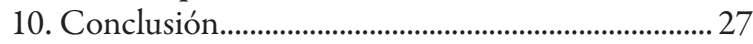

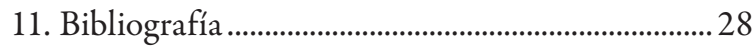

\section{RESUMEN}

Con el gran avance y caudal de cirugía bariátrica y metabólica en los últimos años ofrecida a los pacientes con obesidad mórbida, el cirujano plástico debería estar preparado para poder realizar las reconstrucciones quirúrgicas necesarias de estos pacientes una vez que estabilizan su peso. El presente trabajo hace referencia a las técnicas más usadas en abdominoplastia de los pacientes post-bariátricos, teniendo en cuenta la evalua-

\footnotetext{
1. Rotación Cirugía Estética. Universidad del Salvador

\Correspondencia: Dr. Carlos Matías Luján. matiaslujan81@gmail. com
}

Los autores no declaran conflictos de intereses

Recibido: 06/2018 / Aceptado: 09/09/18 ción prequirúrgica tanto física como el entorno psicológico, asi como la prevención y el estudio de sus posibles complicaciones.

Palabras claves: bariátrica, técnicas, abdominoplastia, complicaciones.

\begin{abstract}
With the great advance and volume of bariatric and metabolic surgery in recent years offered to patients with morbid obesity, the plastic surgeons hould be prepared to be able to perform the necessary surgical reconstructions of these patients once they stabilize their weight. The present work refers to the most used techniques in abdominoplasty of postbariatric patients, taking into account the pre-surgical evaluation both physical and psychological environment, as well as the prevention and study of its possible complications.

Keywords: bariatric, techniques, tummytuck, complications.
\end{abstract}

\section{INTRODUCCIÓN}

Comúnmente, el abdomen, los brazos, los muslos y la región glútea son las áreas de mayor preocupación para los pacientes que han tenido una pérdida masiva de peso luego de una cirugía bariátrica. La apariencia típica de estos pacientes deriva de una combinación de factores, entre los que se incluyen la morfología del cuerpo dependiente del sexo y un cambio importante en el índice de masa corporal (IMC), que conducen a un sobrante de piel con disminución de su elasticidad y tejidos blandos que no se retraen completamente después del metabolismo de la grasa a través de estos tipos de cirugías o después de cambios en el estilo de vida.

El exceso de piel y tejidos blandos descienden inferomedialmente de las áreas de deposición características de la grasa. Las personas obesas suelen tener sobrepeso desde la infancia y casi siempre desde la adolescencia ${ }^{1}$. La edad media para los procedimientos bariátricos es de 37 años ${ }^{2}$. 


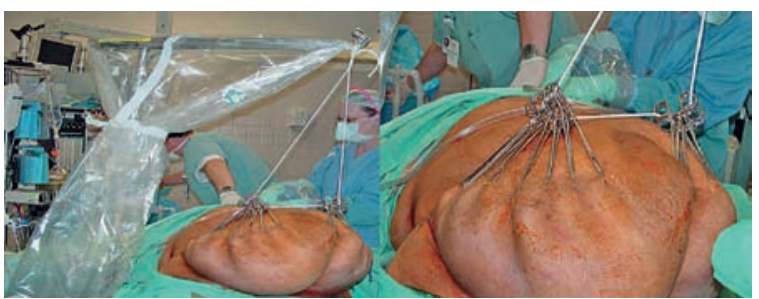

Figura 1. Elevación del panículo adiposo con el dispositivo de suspensión, el cual posee un brazo articulado para la movilización de los colgajos ${ }^{(19)}$.

En los años previos a los procedimientos de reducción gástrica, los individuos ganaban y perdían peso varias veces a través de dieta o la modificación del estilo de vida. Esta historia de pérdida y ganancia de peso en forma alternativa durante años es la queconduce a la disminución de elasticidad de la piel después de la pérdida masiva de peso ${ }^{3}$.

Las deformidades extremas del contorno corporal que distinguen al paciente común de aquel que ha perdido una cantidad masiva de peso han llevado al desarrollo de técnicas quirúrgicas específicas para estos individuos.

Las metas de estas técnicas son: permitir la extirpación del exceso de piel y grasa, corregir la diástasis de los músculos rectos abdominales y reparar hernias si estas están presentes. En los pacientes con abdominoplastia tradicional, el tercer objetivo es tener mínimas cicatrices. Este no es el caso de los pacientes con pérdida masiva de peso.

El contorno es un objetivo más importante que el mínimo de cicatrices en esta población, y varias cicatrices pueden ser necesarias para dar al paciente el contorno corporal deseado 4 .

Las técnicas quirúrgicas más utilizadas en los pacientes con pérdida masiva de pesos que se utilizan para la corrección de las deformidades a nivel abdominal de estos pacientes son: la paniculectomía, la dermolipectomía circunferencial, el levantamiento corporal, la abdominoplastia en flor de lis o la combinación de ambas'.

La paniculectomía y la abdominoplastia se han utilizado indistintamente para describir procedimientos quirúrgicos que eliminan el exceso de piel y grasa de la pared abdominal. Pero se debe hacer una distinción entre estos dos conceptos; la paniculectomía describe procedimientos que eliminan sólo la piel y la grasa, es decir, una operación funcional que elimina un delantal sintomático de la piel, mientras que la abdominoplastia se refiere no solo a la eliminación de la piel y la grasa sino también al tratamiento de los músculos de la pared abdominals.

\section{OBJETIVO}

El objetivo esta monografía es realizar una revisión bibliográfica y detallada de las técnicas más utilizadas para el tratamiento del abdomen en pacientes con pérdida masiva de peso luego de una cirugía bariátrica.
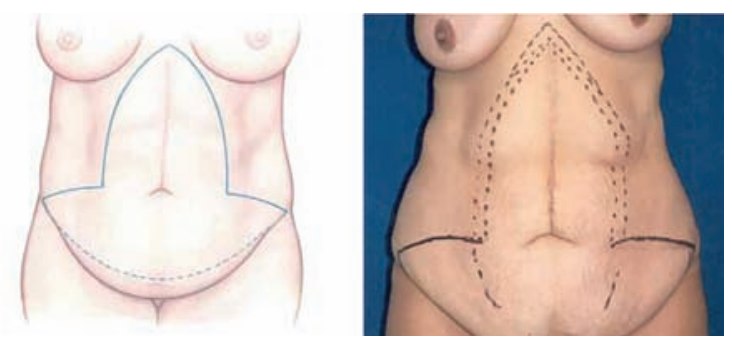

Figura 2. Marcación flor de lis, vista frente y perfil. Los vectores de tracción son el punto fundamental de la técnica(30).

\section{OBESIDAD Y TRATAMIENTOS QUIRÚRGICOS}

La obesidad es una enfermedad en un porcentaje alto de la población mundial. A estos pacientes les toca vivir con un cuerpo de un peso y un volumen excesivo que, aparte de los problemas de salud que les genera, está el agravante de encontrar frente al espejo una imagen que no es la que hubieran deseado tener, generando problemas de discriminación y autodiscriminación, disminución de su autoestima, la dificultad para movilizarse, para hacer deportes y para relacionarse de la misma forma con el resto de la población. La insatisfacción y la tristeza de no saber por qué les tocó a ellos padecer este flagelo los lleva a encerrarse en sí mismos, encontrando un rincón de placer en la comida, que les genera, a su vez, más culpa y frustración por no poder hacer algo para revertir esta situación.

En la actualidad se ha logrado que la misma sea considerada una enfermedad, por ende, es incluida en los programas de salud como cualquier otra patología. Gracias a esto hoy contamos con una amplia gama de tratamientos, al alcance de todos, que le permitirán recibir la ayuda adecuada para poder luchar y terminar con este padecimiento ${ }^{6}$. Así, la cirugía bariátrica se considera el tratamiento de elección si el paciente no es capaz por sus propios medios realizar una pérdida de peso adecuada, lo ideal sería que estos pacientes consulten con su médico y se asesoren para poder recurrir a un equipo multidisciplinario especializado en cirugía bariátrica que cuente con especialistas en metabolismo, cardiología, programas de prevención, gastroenterología, nefrología y hepatología entre otros,que le permita ofrecer atención a los distintos problemas asociados con la obesidad, con la ventaja de centralizar la historia clínica en un solo lugar ${ }^{7}$.

Sin embargo, junto con los enormes beneficios que reciben los pacientes luego del gran esfuerzo por lograr el peso adecuado quedan las "huellas" de ese gran volumen corporal; estas son los colgajos de piel y grasa redundantes que afectan a casi todas las regiones del cuerpo ${ }^{6}$.

Si bien esto sucede en todos los pacientes que han perdido grandes cantidades de peso, la localización 

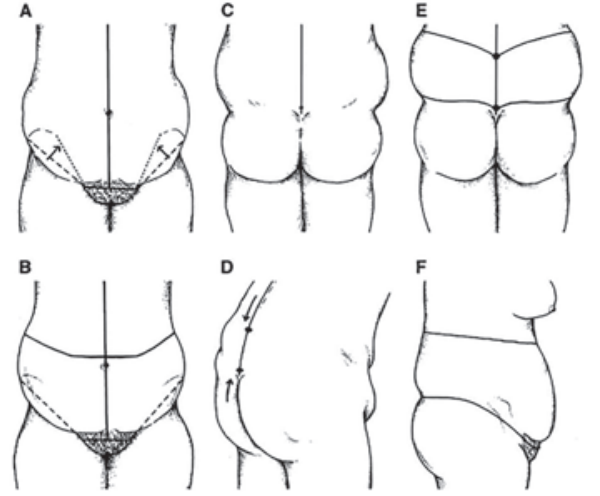

Figura 3. Marcación de abdominoplastia circunferencial. A. La línea media está marcada, y la incisión púbica horizontal está marcada debajo de la línea de implantación pilosa para permitir la elevación de un pubis ptósico. B. Se marca la extensión superior de la resección anterior, y la posición final de la marca de la región inguinal se observa debajo de las espinas ilíacas anterosuperiores. C. Se marca la línea media dorsolumbar. D. La marca de la línea media superior se realiza usando la técnica de pellizco representada por las flechas. Esta maniobra es importante en la prevención de las dehiscencias. E, F. El resto de las marcas laterales y posteriores ${ }^{(4)}$.

de estos colgajos de piel y tejido graso es diferente en las mujeres y en los hombres. Las mujeres tienden a tener exceso de piel a lo largo de la pared abdominal anterior, los flancos y la región de la cadera, también presentan celulitis y exceso de piel a lo largo de los muslos y región glútea.

En los hombres los cambios son similares en las regiones del abdomen, flancos, cadera, muslo medial y regiones púbicas. Junto con los problemas de exceso de piel y tejido blando, el paciente que ha sido sometido a cirugía bariátrica tiende a tener deficiencias en el tono de la piel ${ }^{1}$.

Esto ha impulsado un rápido aumento en el número de pacientes que se presentan a la consulta del cirujano plástico para procedimientos de contorno corporal.Las personas que buscan el asesoramiento y la experiencia de un cirujano plástico con respecto a la eliminación del exceso de piel después de una pérdida masiva de peso han sufrido un acontecimiento importante que altera su vida. Si bien la forma de su cuerpo ha cambiado dramáticamente, quedan estigmas en los colgajos de piel y grasa en las regiones citadas anteriormente. A pesar de la exitosa pérdida de peso, la autoestima puede ser baja. Estos pacientes a menudo afirman que se sienten triplemente estigmatizados: primero por ser obeso mórbido, por elegir la terapia quirúrgica para bajar de peso y por ser considerado frívolo y buscar la ayuda de un cirujano plástico ${ }^{8}$.

Por esta razón es esencial que el cirujano plástico se acerque a estos pacientes de una manera eficiente, teniendo en cuenta que el objetivo de estos tratamientos es devolver la forma y la armonía adecua- da mediante el tratamiento del exceso de colgajos de piel y grasa y de un modelado corporal adecuado ${ }^{6}$.

\section{VALORACIÓN DE PACIENTES POSBA- RIÁTRICOS EN PLAN DE CIRUGÍA DE CONTORNO CORPORAL}

Los puntos clave para una evaluación adecuada del paciente con pérdida de peso incluyen:

- Indice de masa corporal (IMC) prequirúrgico y estabilización del peso.

- Detectar patologías no resueltas asociadas con la obesidad y la cirugía bariátrica.

- Identificar problemas psicosociales relevantes.

- Diagnosticar distrofias luego de la pérdida masiva de peso por cirugía bariátrica.

- Determinar expectativas del paciente.

- Diagramar un plan de tratamiento eficiente y seguro para el paciente.

Si bien la entrevista inicial es un excelente momento para establecer una relación con los pacientes, también es una oportunidad para obtener una historia detallada de su cirugía de pérdida de peso y el cumplimiento con el régimen nutricional. El cirujano debe saber qué tipo de procedimiento tiene el paciente, ya que las diferentes técnicas quirúrgicas en la cirugía bariátrica tienen un variado potencial para causar diferentes déficits nutricionales ${ }^{8}$.

\section{PREMISAS PARA LA SELECCIÓN DEL CANDIDATO A LA CIRUGÍA}

Al momento de decidir realizar una cirugía de contorno corporal en un paciente posbariátrico, el cirujano debe tener en cuenta las cuestiones nutricionales, médicas y psicosociales inadecuadas que puede dar lugar a malos resultados, por lo que cualquier problema que pueda influir en la seguridad del procedimiento debe resolverse antes de la intervención quirúrgica.

La selección del paciente debe centrarse en maximizar la seguridad del procedimiento. Con este objetivo en mente, la aplicación de los siguientes principios son fundamentales:

\section{ESTABILIZACIÓN DEL PESO CORPORAL:}

Los pacientes que se consideran candidatos para la cirugía de contorno corporal deben tener un peso estable durante 3 meses, lo que suele ocurrir entre 12 y 18 meses después de un procedimiento de cirugía bariátrica. Esto es importante en primer lugar porque la homeostasis nutricional y un balance de nitrógeno positivo son necesarios para facilitar el proceso de cicatrización de las grandes heridas quirúrgicas, se puede lograr un resultado más predecible cuando el paciente no está perdiendo peso de manera activa y porque un IMC elevado se asocia con un aumento de las complicaciones de la cicatrización de las heridas ${ }^{13}$. 
ÍNDICE DE MASA CORPORAL ADECUADO:

El candidato ideal para la realización de un procedimiento de contorno corporal es el que tiene un IMC de $28 \mathrm{~kg} / \mathrm{m}^{2}$ o menos, y se debe ser más cuidadoso con el nivel de agresividad de los procedimientos quirúrgicos con aquellos pacientes que tienen un IMC entre 29 $\mathrm{kg} / \mathrm{m}^{2}$ y $32 \mathrm{~kg} / \mathrm{m}^{2}$. En tanto, los pacientes cuyo IMC se encuentra entre 32 y $35 \mathrm{~kg} / \mathrm{m}^{2}$ deben ser seleccionados con gran cuidado, y los procedimientos pueden ser más limitados que para los pacientes con un menor IMC. Algunos pacientes en este rango de IMC pueden beneficiarse de una simple paniculectomía si tal procedimiento mejorara su capacidad de ejercicio y progreso con la pérdida de peso adicional ${ }^{14}$.

\section{NUTRICIÓN ADECUADA:}

El estado nutricional del paciente posbariátrico debe ser óptimo. La mayoría de los pacientes con pérdida masiva de peso tendrán una ingesta de alimentos adecuada para su estado basal. Es raro ver a un paciente posbariátrico con signos manifiestos de desnutrición. Lo que el cirujano plástico debe determinar es si la ingesta nutricional es adecuada para satisfacer las demandas de un procedimiento quirúrgico importante, ya que éstos pueden incrementar los requerimientos nutricionales en un $25 \%$ y muchos de los pacientes con pérdida masiva de peso deberán ajustar sus ingestas. Así, si un paciente tiene una ingesta diaria de proteínas menor a $50 \mathrm{~g}$ no representa un candidato quirúrgico, y la modificación dietética es esencial. Del mismo modo, debido a que muchos de los procedimientos de cirugía bariátrica alteran la fisiología gastrointestinal, muchos de estos pacientes deben tomar suplementos vitamínicos como calcio, vitamina B12 y hierro, por lo que los problemas dietéticos posteriores son de esperar, y los problemas nutricionales deben ser revisados en el período posoperatorio si se presentan complicaciones en cicatrización de las heridas.

También se debe prestar atención si el paciente candidato a la cirugía de contorno corporal tiene síntomas compatibles con una imposibilidad física para comer, se le debe pedir que consulte a su cirujano bariátrico para descartar una estenosis del tracto digestivo ${ }^{8}$.

\section{ESTABILIZACIÓN PSICOSOCIAL Y DE COMORBILIDADES:}

Asi como las comorbilidades médicas de la obesidad se mejoran significativamente con la pérdida masiva de peso, el cirujano plástico debe examinar y buscar recidivas de las mismas, y debe derivar al médico especialista apropiado para que este evalúe al paciente y autorice o no la realización del procedimiento quirúrgico.

En pacientes que son fumadores activos, se les recomienda no fumar al menos 1 mes antes de la cirugía. Si esto no es posible, entonces el alcance del procedimiento realizado, especialmente la canti- dad de tejido a resecar, es limitado. Lo mismo ocurre con los pacientes diabéticos y los tratados con esteroides.

Un equipo de apoyo psicosocial bien establecido es fundamental para la contención de éste tipo de pacientes ya que un paciente bien motivado generalmente representa un mejor candidato para cirugía electiva de contorno corporal. Es de vital importancia este punto para tener el máximo porcentaje de éxito en la cirugía de contorno corporal.

\section{METAS Y EXPECTATIVAS RAZONABLES CONSIDERANDO SU EDAD, SALUD Y HÁBITO CORPORAL}

Los pacientes deben estar dispuestos a aceptar cicatrices extensas a cambio de piel redundante y laxa, comprender las limitaciones de los procedimientos quirúrgicos, como identificar las áreas del cuerpo que no serán modificadas por la cirugía planificada. Este último punto es importante porque la mejora de un área del cuerpo puede resaltar deformidades en áreas adyacentes ${ }^{11}$.

\section{EVALUACIÓN DE DISTROFIAS ABDOMINALES}

Un examen físico exhaustivo deben incluirse en la evaluación inicial del paciente con el fin de identificar todas las deformidades y detectar recidivas de comorbilidades. Se debe evaluar el IMC, el tipo de cuerpo general, el tejido adiposo sobrante, los acúmulos de grasa localizados y pliegues. La distribución de grasa corporal varía mucho en esta población de pacientes y esto influye mucho en las opciones quirúrgicas. Debe prestarse atención al tono y la elasticidad de la piel del paciente.

En el examen abdominal, se debe tener en cuenta el espesor del tejido subcutáneo, la presencia de hernias y eventraciones, diastasis de músculos rectos, laxitud general de la pared abdominal, etc.

Para facilitar el análisis de las deformidades en cada región anatómica del cuerpo,se aplica una escala de cuatro puntos, la escala de las deformidades producidas por la pérdida de peso de Pittsburgh, la que nos sirve como una guía para delimitar la severidad de las deformidades.

Escala de las deformidades producidas por la pérdida de peso de Pittsburgh para la región abdominal:

\begin{tabular}{|c|l|l|}
\hline Puntos & Deformidad & Pocedimiento \\
\hline 0 & Normal & Ninguno \\
\hline 1 & $\begin{array}{l}\text { Piel redundante con míni- } \\
\text { ma o moderada adiposi- } \\
\text { dad sin colgar }\end{array}$ & $\begin{array}{l}\text { Miniabdominoplastia o Ab- } \\
\text { dominoplastia convencio- } \\
\text { nal }\end{array}$ \\
\hline 2 & Panículo colgante & $\begin{array}{l}\text { Abdominoplastia conven- } \\
\text { cional }\end{array}$ \\
\hline 3 & $\begin{array}{l}\text { Múltiples colgajos o gran } \\
\text { adiposidad en epigastrio }\end{array}$ & $\begin{array}{l}\text { Abdominoplastia en flor } \\
\text { de lis o levantamiento cor- } \\
\text { poral superior. }\end{array}$ \\
\hline
\end{tabular}



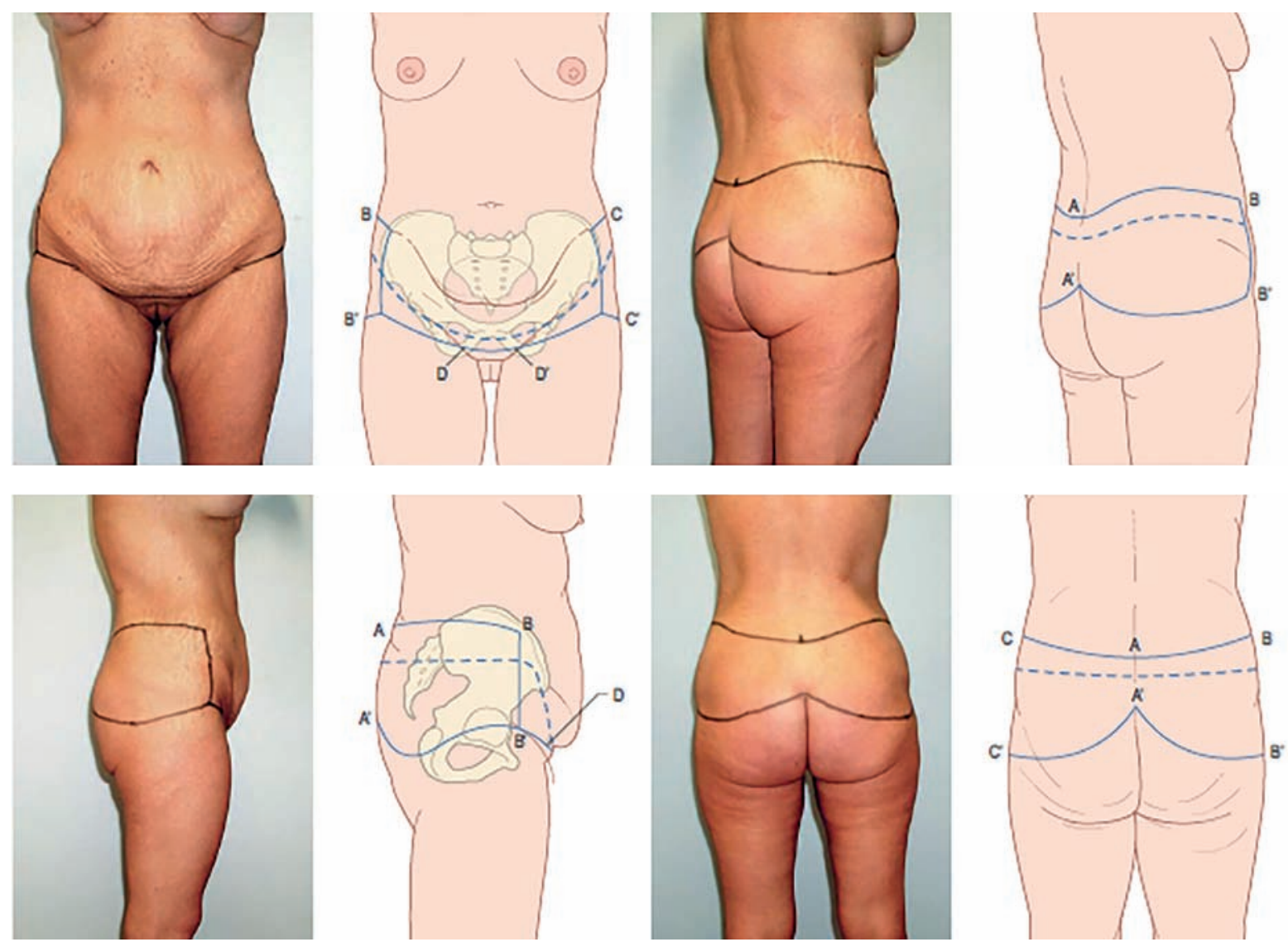

Figura 4. Marcación del levantamiento corporal. La línea punteada indica dónde quedaría la cicatriz final de acuerdo a lo descrito en la técnica(31).

El examen físico del paciente posbariátrico que se presenta para la abdominoplastia debe incluir una evaluación de posibles hernias. Si el paciente ha tenido un procedimiento abierto, existe una alta incidencia de eventraciones. Además, los pacientes que previo a la cirugía bariátrica tenían IMC muy altos a menudo tienen hernias umbilicales. A veces son difíciles de evaluar preoperatoriamente. Es más, si una hernia está presente y cerca del ombligo, debe advertirse al paciente que es necesario sacrificar el ombligo para obtener una reparación óptima de la hernia. El tallo del ombligo en pacientes que antes tenían un alto IMC puede ser muy largo, y en algunos casos podría ser necesario crear un neoombligo en vez de utilizar el ombligo original del paciente ${ }^{12}$.

Varios pacientes con pérdida masiva de peso tienen cicatrices de procedimientos que se realizaron previamente. Las cicatrices más comunes son las cicatrices por encima del ombligo, entre las que se incluyen las cicatrices subcostales resultantes de una colecistectomía abierta. Si se va a utilizar una incisión en la línea media, esta cicatriz no solo se llevará a la parte inferior sino también a la medial y se resecará en parte. Por lo general, esta cicatriz subcostal anterior terminará en el nivel del ombligo. A pesar de este acortamiento de la cicatriz, todavía hay preocupación por la viabilidad de la piel y la grasa inferior a esta cicatriz.
En la mayoría de las veces, tal vez debido al aumento de la vascularización que se desarrolló cuando el paciente era obeso, este tejido puede sobrevivir sin ningún problema. Sin embargo, los pacientes con comorbilidades como los pacientes tabaquistas tendrán un mayor riesgo de pérdida de tejido. Además, incisiones no convencionales se pueden diseñar para incorporar o acomodar las cicatrices abdominales superiores.

En los pacientes que han sido sometidos a un procedimiento bariátrico abierto, la cicatriz de la línea media anterior se utiliza para resecar el exceso de piel y grasa en una dirección horizontal y una vertical abdominoplastia en flor de lis ${ }^{15}$.

\section{TRATAMIENTO DE LA PARED ABDOMINAL}

Es frecuente que el cirujano plástico encuentre en los pacientes posbariátricos una hernia incisional. En estos pacientes, se considera si hubo suficiente pérdida de peso como para evitar ejercer una presión excesiva sobre un compartimento intraabdominal todavía obeso. Si el paciente no ha alcanzado un peso corporal adecuado para la reparación de la hernia, entonces se considera posponer el procedimiento. Las hernias muy grandes pueden requerir una liberación extensa de adherencias y / o separación de los componentes de la pared abdominal para lograr el cierre. Cuando se prevé una reconstruc- 
ción de la pared abdominal, limitamos los procedimientos de contorno corporal a una paniculectomía.

En cambio cuando las hernias son de pequeño o moderado tamaño, se combinan la reparación de estas con los procedimientos de contorno corporal en el mismo tiempo quirúrgico ${ }^{16}$.

Así durante el procedimiento quirúrgico una vez que se diseca y libera el colgajo dermograso, tanto las hernias y diástasis de los rectos se pueden tratar. Una técnica que ha tenido mucho éxito en estos pacientes implica una reparación de la hernia sin abrir el saco de la hernia y utilizando una malla de material reabsorbible. El saco de la hernia se diseca libremente sin abrirlo se invagina su contenido y luego la reparación de la hernia se realiza mediante imbricación primaria de la fascia. Esto evita posibles complicaciones, como por ejemplo abrir el saco de hernia y entrar en la cavidad peritoneal, con la perforación intestinal $\mathrm{u}$ otras alteraciones intraabdominales. Se utiliza vicryl para realizar la sutura de fijación la cual se la deja larga, para luego hacerla pasar a través de ella una malla que se fija sobre ésta. Para finalizar se termina de fijar la malla con puntos separados de Vicryl.

El ombligo se lleva a través de una hendidura en la malla. Si la hernia envuelve el ombligo, el ombligo es amputado, y se puede reconstruirlo realizando un neombligo. Debajo de la hernia, todavía habrá una diastasis de los músculos rectos abdominales que debe ser reparada. En los pacientes sin hernia, la imbricación de los bordes mediales de los músculos rectos abdominales debe realizarse. Se han propuesto varias técnicas. Debido a la gran laxitud con la que se presentan las paredes abdominales de los pacientes posbariátricos, algunos cirujanos optan por una imbricación en doble capa, primero haciendo una imbricación estándar, y luego una de refuerzo para apretar la hernia de nuevo y ajustar adecuadamente la fascia muscular ${ }^{17}$.

\section{TRATAMIENTO DE LAS DISTROFIAS ABDOMINALES}

Los pacientes que pierden peso luego de la cirugía bariátrica, comienzan a desarrollar piel excedente que sobresale en muchas áreas de su cuerpo. Generalmente, el abdomen es un área focal principal de la preocupación en pacientes con pérdida masiva de peso. Se han descrito varias técnicas para el tratamiento de ésta área en particular. Las metas de todas estas técnicas son: permitir la extirpación del exceso de piel y grasa, corregir la diastasis de los músculos rectos abdominales y/o reparar hernias si están presentes. En los pacientes a los que se realizan abdominoplastias tradicionales, el tercer objetivo es tener cicatrices mínimas. Este no es el caso de los pacientes posbariátricos. Lograr un buen contorno corporal es un objetivo más importante que obtener la menor cantidad posible de cicatrices en esta población, y varias cicatrices pueden ser necesarios para dar al paciente el contorno corporal deseado ${ }^{4}$.
A menudo, la paniculectomía puede considerarse un procedimiento cosmético, mientras que una abdominoplastía se refiere a un tipo de operación más reconstructiva. Una paniculectomía se puede hacer en pacientes que aún no han comenzado su pérdida de peso para quitar un gran delantal abdominal de piel y grasa, o en los pacientes que tienen un gran delantal abdominal de piel y grasa que sobresale mucho más, después de la pérdida masiva del peso y tienen interferencia con actividades de la vida diaria o bien un historial de patologías dermatológicas recurrentes. Para el paciente con pérdida masiva de peso, una abdominoplastia se hace comúnmente después de que se completa la pérdida de peso, y se realiza para reconstruir la pared abdominal con la eliminación de exceso de piel y grasa, así como el tratamiento de la pared abdominal.

Como regla general, se puede prestar más atención a los objetivos estéticos a medida que disminuye el IMC del paciente 5

Las complicaciones de la herida tienden a ser mayores cuando las operaciones de contorno corporal se realizan en pacientes que siguen siendo obesos, y un procedimiento más agresivo, puede suponer un mayor riesgo de secuelas locales e incluso sistémicas.

Una dermolipectomía en cinturón se refiere a una resección circunferencial de piel y grasa que a menudo también incluye el tratamiento de la pared abdominal dentro de la misma cirugía. Los pacientes que se han sometido a un procedimiento abdominal, ya sea una abdominoplastia o una paniculectomía, pueden optar por someterse a una dermolipectomía en cinturón en un momento posterior. En estos pacientes, la resección se inicia en la región posterior y las orejas de perro son extirpadas anteriormente, revisando así la cicatriz de la porción abdominal de su procedimiento anterior.

Los profesionales que proponen la dermolipectomía en cinturón o el levantamiento corporal como procedimientos iniciales sostienen que el exceso de piel y grasa a nivel lateral puede ser acentuado si se realiza solamente una abdominoplastía como procedimiento inicial.

La resección del panículo graso abdominal se limita solamente al abdomen anterior, pero no abordará áreas tales como exceso de grasa en el área posterior de la cadera. La evaluación preoperatoria del paciente debe incluir la discusión de la anatomía del paciente, la extensión de la cirugía, y las áreas que no serán abordadas durante esta cirugía. Si el paciente desea que se aborden estas áreas, se deben discutir procedimientos alternativos. Revisar fotos de pacientes con variaciones anatómicas similares puede facilitar la discusión y las expectativas.

La abdominoplastia en los pacientes posbariátricos se puede combinar con otros procedimientos, considerando a cada paciente individualmente y tomando en consideración cuestiones de seguridad tales como: la duración total de la cirugía y la salud general del paciente. 
La marcación para éstos procedimientos de modelado corporal que tienen como punto focal el tratamiento de la región abdominal se realizan en el momento preoperatorio y se inician con el paciente en posición de pie. Evitar las orejas de los perros es crítico, y para lograrlo es clave la marcación del final del panículo graso abdominal. Cuando el paciente se encuentra en decúbito, éste saliente lateral se pierde.

En los pacientes que han sido sometidos a un procedimiento bariátrico abierto, la cicatriz de la línea media anterior se utiliza para resecar el exceso de piel y grasa en una dirección horizontal y una vertical generalmente se utiliza la técnica de abdominoplastia en flor de lis. En cambio en pacientes que han tenido un procedimiento laparoscópico o que han perdido su exceso de peso a través de la dieta y el ejercicio,osea por medidas higienicodietéticas y cambios en el estilo de vida, debe hacerse una evaluación de la redundancia de la piel y la grasa en la parte superior del abdomen para determinar si será necesario la realización de un procedimiento que implique una cicatriz vertical sobre la línea media abdominal. Si hay un exceso de piel y grasa en la parte superior del abdomen, se debe considerar la posibilidad de una cicatriz en la línea media. Las incisiones verticales se han utilizado para abordar la parte superior del abdomen desde 1916, cuando Babcock describió elipses verticales de grasa y piel con amplia disección y aproximación hacia la línea media para contornear la cintura y el abdomen. Si no se utiliza una cicatriz en la línea media, puede quedar una redundancia en la parte superior del abdomen y los pacientes pueden no estar satisfechos en el posoperatorio.

El objetivo debe ser la eliminación de la mayor cantidad de piel y grasa en lugar de preocupación por las cicatrices. Puede ser necesaria una mezcla de cicatrices horizontales y/o verticales para obtener el contorno corporal deseado.

El área abdominal superior también puede ser abordada en una etapa posterior con la adición de una cicatriz de línea media, o incluso, en algunos pacientes, se puede utilizar una cicatriz lateral que continúa de una cicatriz de braquioplastia, dirigiéndose a los pliegues laterales de la mama y a la laxitud residual de la parte superior del abdomen, todo con una incisión. Algunos cirujanos incluso han sugerido una incisión abdominal superior o escisión de "corte de melón" para extirpar el exceso de piel y grasa en el abdomen superior ${ }^{17}$.

\section{A. PANICULECTOMÍA PREBARIÁTRICA}

Por varias razones, un paciente puede presentarse a un cirujano plástico para la eliminación de un panículo de piel y grasa abdominal extremadamente grande sin haber tenido ninguna pérdida de peso. En algunos pacientes con un gran panículo abdominal sobresaliente que impide la deambulación y dificulta la higiene, se puede combinar la cirugía bariátrica con paniculectomía a pesar de que la experiencia nos muestre que exis- te una tasa de complicaciones muy alta al combinar estas dos cirugías.

En general se tiende a hacer primero la paniculectomía y permitir así, que el paciente se recupere completamente antes de proceder a realizar la cirugía bariátrica. Pero algunos pacientes con obesidad mórbida requerirán la extirpación de su gran panículo de piel y grasa abdominal para poder permitir un mejor acceso a la cavidad abdominal y así lograr el tratamiento de otras patologías quirúrgicas como histerectomía para el tratamiento del cáncer uterino, o el tratamiento quirúrgico del cáncer colorrectal ${ }^{18}$.

El peso del panículo abdominal puede hacer que la disección quirúrgica sea difícil y también conducir a una pérdida sanguínea significativa. Además, la dificultad de operar en la región que se encuentra debajo del panículo abdominal puede aumentar el riesgo de infección de la herida en pacientes que en su gran mayoría ya tienen un mayor riesgo de infección debido a otras comorbilidades. Por estas razones, el uso de un sistema de suspensión del panículo abdominal durante la cirugía puede ser útil ${ }^{19}$. La extensión lateral del panículo abdominal se debe marcar preoperatoriamente con el paciente de pie.

Con el paciente ya en la mesa de operaciones y bajo anestesia general, se realiza la antisepsia con solución de yodopovidona y se colocan los campos quirúrgicos. El dispositivo de suspensión del panículo abdominal, se cubre entonces con un campo estéril y se colocan a lo largo de toda la extensión del panículo abdominal. De esta manera el dispositivo de suspensión se eleva y queda así suspendido el panículo abdominal (Figura 1).

La disección se inicia entonces desde los bordes más laterales del panículo abdominal y se llega en profundidad hasta la fascia; éste nivel la disección se continúa hacia la línea media. Esta puede ser llevada a cabo por dos equipos.

A medida que avanza la disección, el dispositivo de suspensión que sostiene el panículo abdominal se eleva elevando el panículo adiposo de la pared abdominal y ayudando a delinear el plano de disección deseado a nivel fascial. Esta elevación tiene como efecto drenar parte de la sangre del plano de disección y además aumentar la visibilidad del plano quirúrgico.

Debe tenerse cuidado al acercarse a la región del ombligo, ya que algunos pacientes pueden tener una hernia umbilical que puede no haber sido palpable antes de la cirugía debido al gran tamaño del panículo abdominal del paciente. El ombligo suele amputarse durante este procedimiento. El riesgo de infección aumenta en los pacientes con obesidad mórbida, a pesar de esto, algunos cirujanos reportan índices de cierres de heridas exitosos y tasas de infecciones aceptables. Pero en otros la experiencia ha sido diferente y por lo tanto se ha desarrollado una técnica de manejo de heridas abierta para minimizar el riesgo de infección. Se colo- 
can puntos de colchonero grandes con nylon 2-0 a intervalos de aproximadamente 6 pulgadas. Para el confort del paciente, es preferible poner el nudo de la sutura por encima de la incisión para facilitar la extracción de puntos posteriormente.

Dado que estos pacientes suelen ser obesos mórbidos, puede ser difícil obtener una camilla que pueda soportar el peso de éste tipo de pacientes, por lo que la extracción de los puntos a veces se hace con el paciente en una silla de ruedas o sentados.

La herida se cubre con una gasa Kerlix húmeda que se cambia dos veces al día, y los puntos se retiran a partir de la segunda semana. Esta técnica se ha utilizado con éxito tanto para pacientes antes de cirugía bariátrica como en pacientes que requieren histerectomía o cirugía intestinal. Aunque esta población de pacientes pueden ser algunos de nuestros pacientes más felices, hay algunos factores que deben tenerse en cuenta para maximizar el resultado. Uno de los más importantes es evitar las orejas de perro. Marcar al paciente en posición de pie para delinear la extensión lateral del panículo graso abdominal sobresaliente minimizará este problema. La incisión abdominal inferior es mucho más larga en los pacientes post pérdida masiva de peso que en otros pacientes que se presentan para una abdominoplastia convencional ${ }^{20}$.

También es importante resecar una porción del área superior del monte Venus, si esta es laxa. A menudo un paciente al que se le ha realizado una paniculectomía y se le ha dejado con un monte Venus redundante puede no quedar satisfecho con el resultado. Por lo general se reseca el monte Venus en forma horizontal a una distancia de alrededor de tres dedos por encima de la hendidura labial. La resección del monte Venus conducirá a un aumento riesgo de drenaje linfático el que debe ser evitado. La decisión sobre cuánto monte Venus se deberá resecar se hace sobre la mesa de operaciones, ya que puede ser difícil elevar el área bajo el panículo adiposo abdominal mientras el paciente está de pie ${ }^{21}$.

La laxitud recurrente es un problema en cualquier paciente después de una pérdida masiva de peso. No importa cuanta piel se reseque, se puede esperar que con el tiempo se relaje, lo que lleva a una cierta recurrencia del defecto. El abdomen superior es un área donde la laxitud recurrente puede ser particularmente molesta para el paciente. Los pacientes están más dispuestos a cambiar el contorno que a evitar las cicatrices, y la posibilidad de una incisión de la línea media debe ser considerada. En algunos pacientes, también se puede utilizar una excisión lateral, especialmente como continuación de una incisión de braquioplastia en pacientes con laxitud lateral a su zona de mama.

El riesgo de seromas es mayor en esta población. La grasa parece diferente en estos pacientes, es evidente que todavía hay demasiadas células de grasa presentes. Se recomienda el uso de cuatro drenajes para dre- nar adecuadamente el área. Incluso entonces, algunos pacientes pueden desarrollar un seroma ${ }^{22}$.

\section{B. ABDOMINOPLASTIA EN FLOR DE LIS}

La abdominoplastia en ancla evolucionó a partir de 1909 con Weinhold, pasando por Somalo en 194024, Duffourmentel en 1959, Castañares en 1967(25) y Regnault en 1975 con el diseño de Flor de Lis ${ }^{26}$, hasta la fecha actual con la utilización del abordaje multifuncional propuesto en $2002^{27}$. Con esta técnica, realizada en un solo tiempo quirúrgico, se pretende tratar no solo el abdomen en delantal, sino también el contorno corporal como un todo, a través de una resección en monobloque de la región abdominal, preconizado por Pontes ${ }^{28}$ sin despegamiento de los colgajos remanentes, lo que genera vectores oblicuos que modelan el dorso y los flancos y elevan el púbis y el tercio superior de los muslos; todo ello con una menor pérdida sanguínea y una mayor seguridad vascular de los colgajos ${ }^{29}$.

Marcación

Con el paciente en posición ortostática marcamos un punto de inicio sobre el apéndice xifoides (Punto A). De este punto parten dos líneas cóncavas en relación al ombligo, en dirección a las líneas hemiclaviculares, descendiendo hasta un punto que se determina mediante maniobras bimanuales semejantes a las empleadas en la técnica de mamoplastia de reducción mamaria de Pitanguy (puntos $\mathrm{B}$ y C) ${ }^{27}$ por tracción oblicua, normalmente situados $2 \mathrm{~cm}$ debajo del ombligo. Marcamos un punto suprapúbico (aproximadamente a $1 \mathrm{~cm}$ del pubis), en la línea media (punto D), que varía de acuerdo a la flaccidez del paciente. Trazamos una línea desde la región suprapúbica hasta la cresta ilíaca, paralela al pliegue inguinal. Unimos los puntos B y D manualmente, diseñando una elipse que irá a encontrarse con la línea ya trazada en la región inguinal. Realizamos la misma maniobra en el lado contralateral (puntos $\mathrm{C}$ y D), se consigue de esta forma el diseño correspondiente a la resección quirúrgica en monobloque (Figura 2).

\section{Cirugía}

Con el paciente en la mesa de operaciones y bajo anestesia general, se realiza la antisepsia de la región abdominal con solución de yodopovidona y se colocan los campos quirúrgicos. Se incide la piel en toda el área previamente marcada. Se inicia la disección del monobloque a partir del punto $\mathrm{A}$, de forma cráneo-caudal y latero-medial con el objetivo de utilizar el propio peso del colgajo para facilitar la retirada de la pieza. Es importante enfatizar que la disección latero-medial permite abordar los defectos herniaros de la pared abdominal a partir de una aponeurosis sana, en dirección al saco herniario, conjugando de esta manera una disección agresiva y a la vez segura. La disección se realiza con bisturí eléctrico para disminuir drásticamente el sangrado intraoperatorio. El tratamiento del ombligo debe tener en cuenta: el aumento circunferencial poso- 
peratorio del mismo; la posición del ombligo resultante y la longitud del pedículo umbilical.

Para solucionar el aumento circunferencial del ombligo, se reducen sus dimensiones al mínimo tamaño posible. La tendencia de la posición del ombligo en estos pacientes es a ser baja, por eso se debe ubicar el nuevo ombligo a unos $4 \mathrm{~cm}$ por encima de su posición inicial, lo que hace el aspecto del abdomen más elegante.

En cuanto a la longitud del pedículo umbilical, se amputa, de manera que quede armónico como en la abdominoplastia clásica. La plicatura de la aponeurosis debe ser agresiva, ya que estos pacientes han sufrido una pérdida significativa de grasa intraperitoneal. Para su realización recomienda la utilización de puntos en "X" con vicryl 0.

En estos pacientes puede ser necesaria la utilización de una malla Marlex luego de la plicatura, ya que estos pacientes habitualmente presentan una gran flaccidez músculo aponeurótica. Se inicia la plicatura de superior hacia inferior, interrumpiéndola $5 \mathrm{~cm}$ por encima del ombligo y desde el pubis hacia arriba, con el propósito de favorecer la ascensión del pedículo umbilical, que luego deberá ser fijado a la aponeurosis con puntos simples de mononylon 3.0. En esta etapa se lleva a cabo el tratamiento del pubis con puntos de vicryl 2.0 ( 3 a 5 puntos), buscando elevar todas las estructuras relacionadas con él y mejorando significativamente la estética genital. Con esta técnica descartamos la indicación de resección de piel en esta región.

Se sutura un punto central suprapúbico uniendo los puntos B, C y D con vicryl 0 o mononylon 0 . Se realizan puntos de distribución con nylon 3.0 para evitar correcciones posteriores, y para el resto de la sutura, se hace en tres planos con monocryl 3.0 y 4.0 , y con nylon 3.0. La sutura de piel debe ser reforzada con puntos simples de nylon 4.0 dada la alta incidencia de problemas cicatriciales (estos puntos serán retirados más tarde).

La sutura de la cicatriz umbilical debe hacerse directamente sobre los bordes de la incisión previa, sin necesidad de resecar la piel adicional, dada la tendencia al aumento de diámetro circunferencial. Luego se confecciona un vendaje con compresas que inmoviliza toda el área abdominal y que se retira al dia siguiente, colocando entonces una cinta modeladora ${ }^{30}$.

\section{ABDOMINOPLASTIA EN CINTURÓN (ABDOMI- NOPLASTIA CIRCUNFERENCIAL)}

La combinación de flancoplastia con la abdominoplastia en una única etapa está limitada a los pacientes con laxitud cutánea específicamente en flancos con depósitos de grasa o sin ellos, en los que la liposucción no puede ofrecer buenos resultados. Además incluye los beneficios otorgados por cada procedimiento por separado, en el caso de la abdominoplastia se mejora a nivel abdominal la laxitud de la piel, el exceso dermograso y la flaccidez muscular. Y la flancoplastía permite re- ducir el volumen de los flancos, levantar o reducir la región glútea al incluir el volumen del tercio superior de éstas y mejorar la laxitud cutánea de los flancos.

Constituye un procedimiento seguro si se ejecuta criteriosamente y los riesgos o complicaciones son los mismos que al ejecutar los procedimientos por separado.

Los pasos quirúrgicos incluyen la marcación del exedentecutáneograso en los flancos con la paciente de pie, sin realizar marcación sobre el abdomen (Figura 3).

Ya que la flancoplastia comprende una amplia resección fusiforme de la piel y grasa subcutánea, en profundidad hasta la aponeurosis muscular, a lo largo del eje mayor de los flancos, tomando como referencia las espinas ilíacas anterior y posterior, la mejor forma para estimar la magnitud de la resección es la prueba del pellizco, con la paciente de pie. La marcación debe adoptar una configuración fusiforme, ubicada a unos pocos centímetros de las crestas ilíacas. En la región sacra la marcación se puede realizar de dos formas diferentes. En los casos que requieren de una cantidad limitada de resección, la marcación posterior puede detenerse a diferentes distancias de la línea media posterior. Cuando la marcación se une con la del lado opuesto, en el extremo superior del surco interglúteo, queda delimitado un área de piel adicional a éste nivel en forma de "V" abierta hacia cefálico. Esto permitirá durante la cirugía acomodar mejor la piel y evitar la formación de orejas de perro haciendo innecesaria la lipoaspiración secundaria ${ }^{4}$.

\section{Cirugía}

La cirugía comienza con la paciente en posición prona y la realización de la flancoplastia que consiste en la disección del área previamente marcada hasta la aponeurosis muscular en profundidad, sin realizar ningún despegamiento extra. La herida se cierra por planos sin dejar espacios muertos. El tejido celular subcutáneo con vicryl 2-0, dermis con vicryl 3-0 y una sutura intracuticular con vicryl 4-0. La línea de sutura se cubre con steristrip. Hacia los laterales queda una cantidad de exceso cutáneo dispuesto como orejas de perro. Luego se rota al paciente en posición supina y se realiza la abdominoplastia independientemente de la flancoplastia en todas sus etapas. El exceso de piel de los flancos y el abdomen se diseca sin realizar disecciones adicionales, excepto para ajustar los colgajos a efectos de acomodarlos para que se unan en una línea de sutura armoniosa en forma de manubrio de bicicleta con los flancos.

Las prominencias, la región superior, media y anteroinferior de la pared abdominal constituyen problemas secundarios comunes. La queja más común es que antes de la cirugía no tenían abdomen protruido, siendo esto una falsa percepción. Después de la abdominoplastia, los segmentos abdominales medio y bajo quedan retruidos con lo cual se expone el problema pre- 
existente. Puede observarse prominencia del hipogastrio asimétrico porque existía previamente un depósito de grasa voluminoso a un costado. Otro detalle es el escalón del pubis a lo largo de la cicatriz después de dos a tres meses. Esto se explica por el edema residual no reabsorbido por el tejido graso. Para esto lo mejor es la liposucción.

En el ombligo pueden producirse problemas secundarios. La necrosis y estenosis del pedículo se tratan con neoumbilicoplastia. Se reseca el ombligo residual y esta área cura por segunda intención. La mala posición se reemplaza por una posición correcta, fijándolo a la aponeurosis. La cicatriz remanente es menos evidente que el ombligo en posición incorrecta.

La lipoaspiración de las caderas constituye un procedimiento de rutina para lograr la simetría estética.

La abdomino-flancoplastia está indicada en flancos fláccidos y cuando la liposucción no puede ofrecer buenos resultados 5 .

\section{LEVANTAMIENTO CORPORAL}

El procedimiento de contorno corporal ideal para el paciente que ha perdido una enorme cantidad de peso debe abordar eficazmente todos o la mayor parte de los estigmas característicos de una manera segura, eficiente y consistente. Se han descrito varias técnicas para tratar la condición corporal postbariátrica; estos incluyen elevación del cuerpo y lipectomía del cinturón. Aunque tienen nombres diferentes, cada uno implica una abdominoplastia y levantamiento de muslo y glúteos en forma simultánea.

El objetivo de todos estos procedimientos es revertir el colapso inferomedial de la piel y los tejidos blandos de la parte inferior del cuerpo. Aparte de la ventaja evidente de abordar los muslos y la región glútea, así como el abdomen en una sola etapa, un procedimiento circunferencial simultáneo ofrece otra ventaja muy importante: la resección del excedente dermograso no está limitada por la longitud de la cicatriz. En cualquier procedimiento que está limitado por la longitud de la cicatriz, se debe tener precaución en el grado de tracción de la piel para evitar la redundancia de piel a nivel medial y lateral de la cicatriz. Los procedimientos circunferenciales permiten niveles mucho más altos de tensión que se aplicarán sin esta precaución. Esta es particularmente importante para lograr el levantamiento distal de los muslos y el abdomen superior al realizar un abordaje desde la cintura.

La selección de los pacientes y la preparación adecuada antes de la cirugía son esenciales para maximizar la probabilidad de lograr un buen resultado y minimizar las complicaciones. Los pacientes deben tener un peso estable durante varios meses e idealmente deben estar en su peso más bajo antes de la cirugía.

La desventaja de realizar procedimientos de contorno corporal en pacientes con pérdida de peso en curso, es el potencial de recurrencia temprana de la laxitud del tejido. Debe evitarse realizar levantamientos corporales en individuos con un IMC superior a $35 \mathrm{~kg} / \mathrm{m}^{2}$; el levantamiento circunferencial en esta población tiene un efecto mínimo sobre el exceso de piel y celulitis a nivel de los glúteos y los muslos. En ocasiones, se puede ofrecer levantamientos corporales a este grupo más pesado de pacientes a aquellos menores de 35 años, generalmente hombres, pero también mujeres que tienen una distribución de grasa más central. Evitamos realizar un levantamiento corporal en un paciente que ha sido sometido a una cirugía bariátrica y que son mayores de 55 años. Las personas con obesidad mórbida que han buscado cirugía bariátrica en la quinta y sexta décadas de la vida a menudo han desarrollado artritis degenerativa y, en muchos casos, se han sometido a un reemplazo articular y la recuperación del levantamiento corporal, en estos pacientes, resulta difícil y prolongada.

Las anemias tienden a ser secundarias a la mala absorción de hierro y ácido fólico. Se recomienda a los pacientes que estén considerando levantar el cuerpo que tomen un suplemento de hierro y multivitaminas diarias. Los pacientes gravemente anémicos son derivados a un hematólogo. Preferimos una hemoglobina de línea base de 12 .

\section{Premisas quirúrgicas}

La marcación cuidadosa del paciente antes de levantamiento corporal es esencial para un resultado óptimo. Los procedimientos de contorno del cuerpo circunferencial tienen el objetivo común de minimizar la perceptibilidad de la cicatriz colocando la cicatriz en un nivel fácilmente oculto por la ropa. Un análisis de la ubicación en la que los hombres y mujeres usan sus pantalones, ropa interior, trajes de baño, etc., revela que la parte superior de la mayoría de las prendas de vestir se encuentran en la región de la cadera a nivel de las espinas ilíacas anterosuperiores (EIAS) o aproximadamente 6 a $7 \mathrm{~cm}$ por debajo del borde superior de las crestas ilíacas.

En la región del dorso, las prendas se ubican horizontalmente a lo largo de la espalda baja y por encima de los glúteos, también al nivel de las EIAS. En la región anterior, prácticamente todas las prendas interiores cubren la interfaz entre el área del vello púbico y el hipogastrio. Lo ideal es que la cicatriz del levantamiento corporal se localice a nivel de las EIAS a lo largo de la cadera y la espalda baja, y descienda gradualmente a la interfaz entre el área del vello púbico y el hipogastrio en la región anterior. Una técnica efectiva para marcar el levantamiento corporal debe producir una cicatriz que se encuentre confiablemente a lo largo del nivel de estos puntos de referencia, a pesar de la movilidad extrema del tejido del paciente con pérdida masiva de peso y el alto nivel de tracción requerido para lograr un cambio significativo. Para ello, se requiere una técnica de marcado que use puntos de referencia óseas, como las EIAS, para controlar la localización de la cicatriz. 
Marcación

Con el paciente de pie, se marca un área por encima de la línea glútea, que traccionando la piel hacia abajo debe quedar a nivel de las EIAS (punto A). A menudo, las EIAS son difíciles de palpar, pero generalmente se encuentran aproximadamente tres dedos $(6-7 \mathrm{~cm})$ por debajo de las crestas ilíacas. Luego se marcan a nivel de ambas líneas axilares anteriores, derecha e izquier$\mathrm{da}$, dos puntos, que traccionando la piel hacia abajo deben quedar a nivel de las EIAS punto B derecho y $\mathrm{C}$ izquierdo.

Traccionando la piel hacia abajo sobre los glúteos y muslos, se unen con líneas de puntos A, B y C. La línea de puntos con tracción hacia abajo debe estar alineada con la cintura del paciente. Con tracción hacia debajo de los glúteos y muslos, el resultado debe ser una línea de puntos recta que se extiende del punto $\mathrm{B}$ a la derecha al punto $\mathrm{C}$ de la izquierda, que pasan por el punto A sobre la hendidura glútea.

Por maniobra de pinch test se marcan los puntos A', B' y C'. La piel redundante de los glúteos y muslos se estima entonces con la técnica de pinch test. Los puntos B-B' y C-C' se llaman puntos de compromiso ya que el cirujano no vuelve a medir la distancia entre estos puntos durante la cirugía y se compromete a remover el tejido según la marcación prequirúrgica. El punto A' es sólo estimativo.

Con el paciente acostado en posición supina y horizontal en la cama, y la tracción de la piel anterior hacia arriba, se marca una línea tranversal sobre la región púbica (puntos D y D'). Esta línea se coloca aproximadamente $6 \mathrm{~cm}$ por encima de la comisura vulvar anterior o de la base del pene. Traccionando el abdomen hacia arriba, se marca una línea recta desde D a B', y de manera similar de D’ a C'. Esto permitirá la corrección del exceso de piel de la región anterior y medial del muslo. En los pacientes con exceso de piel moderado a severo, las líneas de D-B' y D-C' recaerán en los muslos cuando no se realiza la tracción (Figura 4).

\section{Cirugía}

En el quirófano, con el paciente de pie se realiza la antisepsia desde los hombros hasta los tobillos con solución de yodopovidona. Luego, el paciente se sienta en una mesa de operaciones previamente cubierta con campos estériles y se ubica en decúbito ventral y horizontal. Se colocan medias y dispositivos de compresión secuencial estériles. Previamente se coloca una zalea en el sector medio del campo operatorio. Después de la anestesia endotraqueal general, se coloca un catéter de Foley y se terminan de colocar los campos quirúrgicos.

Se incide superficialmente la piel de A-B y de A-C $1 \mathrm{~cm}$ por encima del punto A se realiza una incisión superficial y vertical para determinar la línea media. Se incide superficialmente la piel desde B-B', de C-C'y de C'-B'. $\mathrm{Si}$ se va a realizar liposucción de los muslos se hace en este momento. Se realiza infiltración tumescente solamente en los tejidos que van a ser lipoaspirados. Se rota al paciente a decúbito dorsal y se realiza la incisión de la piel y el celular subcutáneo hasta la fascia de C'-B', pasando por el monte Venus donde la incisión se realiza biselada hacia abajo para lograr resecar mayor cantidad de tejido adiposo en ésta zona. Se eleva el colgajo abdominal hasta la altura del ombligo, que se desinserta quedando fijo a la pared abdominal. Se incide la piel y el tejido celular subcutáneo a lo largo de las líneas verticales C-C' y B-B'. Por encima del ombligo la disección se mantiene por encima de los músculos rectos del abdomen hasta el nivel del apéndice xifoides, preservando las perforantes intercostales.

El colgajo abdominal se puede dividir a lo largo de la línea media hasta el ombligo para permitir una mejor exposición de la región xifoidea. La espalda del paciente se eleva aproximadamente $35^{\circ}$ para evitar la laxitud miofascial. Para exponer el plano miofascial durante la plicatura se coloca un retractor para elevar el colgajo dermograso abdominal. Se marca en forma de elipse el área a realizar plicatura desde el apéndice xifoides hasta el pubis. Se realiza la plicatura con prolene 1.0, sutura continua y nudo enterrado.

La plicatura se realiza en dos partes; desde el pubis al ombligo y del xifoifes al ombligo. Se rota al paciente a decúbito lateral izquierdo con ayuda de la zalea colocada previamente. Se coloca la cintura del paciente flexionada aproximadamente $30^{\circ}$ y las rodillas a $45^{\circ}$. Se completa la incisión de B-A y se extiende aproximadamente 10 $\mathrm{cm}$ más allá de $\mathrm{A}$ hacia $\mathrm{C}$. La incisión más allá de la línea media facilita la disección en el área ubicada por encima de la hendidura glútea y permite una determinación más precisa del excedente de tejido en esta región.

Se realiza la disección del colgajo dermograso en el área de la cadera derecha, el muslo y el glúteo en el plano suprafascial. En la región cefálica de la hendidura glútea la disección del colgajo dermograso se realiza conservando cierta cantidad de tejido graso profundo para evitar la depresión de ésta zona en el posoperatorio. La disección se realiza en sentido caudal hasta aproximadamente cuatro dedos por debajo de la línea B'-A'.

En la región del muslo, la disección continúa hasta nivel del trocánter mayor. La disección puede llegar hasta la rodilla en la región anterior y lateral del muslo. La disección se utiliza en mujeres con exceso de piel y celulitis en la región media del tercio distal del muslo. La cintura se flexiona a $90^{\circ}$ para simular la posición sentada. La extremidad inferior derecha se coloca a $30^{\circ}$ con el uso de un retractor. Una almohadilla mantiene separadas las rodillas $30 \mathrm{~cm}$.

La pierna derecha se sostiene colgada del retractor. Se colocan pinzas en los puntos By B'. Se marca el exceso de tejido graso a resecar; con punto fijo en el colgajo que no fue disecado, se tracciona y avanza el colgajo dermograso del muslo y glúteo desecado hasta unir los puntos B-B'. 
A continuación se disminuye la tensión, para permitir que el colgajo se retraiga aproximadamente $1-2 \mathrm{~cm}$ y se marca el colgajo en este punto. Estos centímetros adicionales son importantes para lograr un cierre óptimo. $\mathrm{Al}$ aproximar los colgajos a nivel del punto A se marca el nuevo punto A' según la cantidad de tejido a resecar. El exceso de tejido dermograso se reseca realizando la disección biselada caudalmente. A través de una incisión en la región lateral baja derecha del pubis se coloca un drenaje de $10 \mathrm{~mm}$ y se lleva a la región glútea. Con puntos de sutura nylon 1.0 se aproxima la dermis y el tejido celular subcutáneo. Se realiza cierre por planos con puntos de monocryl 2.0 para el celular subcutáneo y 3.0 para la dermis. La piel resulta redundante a lolargo de la línea de cierre, luego se reduce con la tensión a lo largo de la incisión durante los primeros meses de maduración de la cicatriz. El paciente se rota a decúbito lateral derecho y se realiza el mismo procedimiento realizado en el muslo y glúteo izquierdos. Una vez en decúbito dorsal, se eleva el torso a $35^{\circ}$. Se tracciona el colgajo dermograso y sin resecar el exceso de tejido en este momento, el colgajo se fija a los bordes de la incisión inferior. Se desciende la posición del tordo a una nueva posición horizontal plana. Si es necesario se realiza liposucción de la región epigástrica y se eleva nuevamente el torso a $35^{\circ}$.

Se realiza resección del excedente dermograso del colgajo abdominal y se fija a los bordes de la incisión inferior, con el paciente en posición horizontal y plano. Se determina una nueva posición para el ombligo y se hace una incisión tipo escudo de $1 \mathrm{~cm}$; la misma debe hacerse aproximadamente $0,5 \mathrm{~cm}$ por encima de la posición umbilical correspondiente en la pared abdominal anterior teniendo en cuenta la retracción adicional que conlleva el sistema fascial superficial y la aproximación cutánea profunda en el momento del cierre. El tallo umbilical se fija a la fascia abdominal y la dermis del colgajo con vicryl 3-0.

Se colocan cuatro drenajes en la región suprapúbica. Dos de los drenajes se colocan en cada muslo y dos en la fascia de la pared abdominal. Los drenajes que se abocan a la pared abdominal se exteriorizan en la región medial baja del pubis, los drenajes que se abocan a los muslos se exteriorizan en el pubis entre el drenaje abdominal y el de la región glútea.

La colocación de los drenajes a través del monte Venus y en un determinado orden sirve para varios propósitos: permite que los pacientes no presenten molestias en la espalda y regiones laterales, que son las posiciones de decúbito de reposo de éstos pacientes en el postoperatorio, las cicatrices son menos visibles a este nivel, evita exteriorizar los drenajes a lo largo de la incisión lo que evita la potencial dehiscencia de la herida y al colocar los drenajes en un orden y ubicación específicos permite saber el débito de cada área.

La espalda del paciente se eleva aproximadamente a $40^{\circ}$ y el borde del colgajo abdominal se fija al borde in- ferior del tejido como se describió para el muslo y las nalgas. Con puntos separados de polipropileno 3-0 (Prolene, Ethicon Inc.) se fija el ombligo. El paciente es trasladado a una cama en una posición de silla de playa quebrada a nivel de la cintura y las rodillas, después de la extubación.

\section{E. CUIDADO POSOPERATORIO}

Los pacientes generalmente están restringidos a una cama de hospital hasta la mañana siguiente ${ }^{31}$. La prevención del embolismo pulmonar es de suma importancia. Los anticoagulantes no se usan perioperatoriamente. Algunos cirujanos abogaron por el uso de heparina de bajo peso molecular antes o después del procedimiento, pero no hay un consenso claro en este momento. Los dispositivos de compresión secuencial y la sonda de Foley se dejan hasta que comienzan la deambulación después de tolerar una posición sentada ${ }^{17}$.

Realizamos exámenes posoperatorios de control (proteínas totales y fraccionadas, hierro sérico y hemograma completo), con la intención de optimizar la recuperación de los pacientes. En el segundo día posoperatorio, el paciente suele ser dado de alta siguiendo un estudio Doppler venoso de la extremidad inferior. Los antibióticos se indican hasta que se retiren los drenajes. Se indican también narcóticos orales y los laxantes también. El primer control ambulatorio es a la semana. En esta visita, solo se eliminan los drenajes con una producción inferior a $30 \mathrm{ml}$ en el período anterior de 24 horas.

Como máximo, se eliminan dos drenajes en cada visita y preferiblemente no del mismo lado. Todos los drenajes se eliminan en 5 semanas, independientemente del débito. Los siguientes controles son a las 6 semanas, 3 meses, 6 meses y anualmente a partir de entonces ${ }^{31}$.

\section{F. COMPLICACIONES}

Las complicaciones posteriores al levantamiento corporal son más frecuentes que con los procedimientos tradicionales de contorno corporal, como la abdominoplastia. Esto es lógico, teniendo en cuenta la magnitud mucho mayor de este procedimiento y el grado de deformidad que se debe corregir en la población que ha perdido una gran cantidad de peso. Sin embargo, las complicaciones generalmente son toleradas bien por esta población de pacientes debido a los beneficios funcionales y estéticos, a menudo dramáticos, que se logran con estos procedimientos.

Los pacientes con un IMC máximo más alto antes de la pérdida masiva de peso al igual que aquellos que han tenido los cambios más grandes en el IMC después de la pérdida de peso tienen un mayor riesgo de complicaciones después de un procedimiento de contorneado corporal.

Los pacientes con antecedentes de tabaquismo tienen un riesgo mayor de complicaciones, como la dehiscencia de la piel y la necrosis cutánea. 
Dehiscencia de la piel

En los procedimientos de levantamiento corporal, la dehiscencia de la piel es la complicación más frecuente. Esto se puede atribuir al hecho de que el procedimiento es circunferencial y que se requiere un alto grado de tracción para producir un buen resultado. El sitio más frecuente de dehiscencia es a nivel de la hendidura glútea y las caderas, las dos áreas de mayor tensión que siguen este procedimiento. Al colocar en flexión al paciente durante la cirugía, simulando la posición sentada, se pone en tensión el área baja de la espalda a nivel de la hendidura glútea, lo cual permite medir de forma adecuada la cantidad de tejido a resecar disminuyendo la frecuencia y gravedad de ésta complicación.

Además en el período posoperatorio temprano el tiempo prolongado de reposo en la cama puede producir un cierto grado de isquemia sobre el sacro y el coxis, lo que contribuye a la mala cicatrización de esta área. Aproximar el sistema fascial superficial, como sugirió Lockwood, permite crear cierto grado de redundancia de tejido a lo largo del cierre durante varios meses. Esta técnica disminuye la incidencia de dehiscencia de la herida y mejora la calidad de la cicatriz.

El tratamiento consiste en el cuidado local de la herida, aunque según la magnitud, puede ser necesario un manejo quirúrgico. Los elementos claves para prevenir la dehiscencia de la herida son: una marcación preoperatoria eficaz y fiable, medición intraoperatoria precisa del tejido a resecar, una técnica de cierre que minimice la tensión a lo largo de los bordes de la herida.

\section{Seroma}

Es la complicación más frecuente de los procedimientos de modelado corporal ${ }^{31}$. Para los procedimientos abdominales, aquellos que tienen mayor riesgo de complicaciones posoperatorias son los pacientes que previo a la cirugía bariátrica llevaban el mayor porcentaje de su peso en la zona abdominal.

Estos pacientes, que se describen como con patrón de manzana o patrón masculino de distribución de grasa, tienen la mayor cantidad residual de grasa y piel en la región abdominal. Antes de que estos pacientes se realizaran la cirugía bariátrica, tenían demasiadas células de grasa (hiperplásicas) y eran demasiado grandes (hipertroficas).

Luego de la cirugía bariátrica los pacientes pierden peso a expensas de la grasa contenida en éstas células pero no disminuyen su número, todavía tienen demasiadas células de grasa, aunque son más pequeñas. La piel y la grasa que se resecan contienen muchas células de grasa reducida, pero la piel y la grasa que quedan aún contienen más células de grasa por área que en los pacientes que nunca han sido obesos mórbidos.

Se sabe que las células grasas segregan muchas sustancias, como la leptina y las citoquinas inflamatorias, que afectan la permeabilidad endotelial. La secreción de estas sustancias por esta gran población de células grasas puede conducir al mayor riesgo de formación de seroma en comparación con pacientes sometidos a abdominoplastia sin pérdida de peso masiva ${ }^{17}$.

La formación de seroma sigue siendo una complicación frecuente después de los levantamientos corporales en la población post-bariátrica. La mayoría de los seromas se ubican en los muslos y, en algunos casos, se pueden extender a las nalgas o la pared abdominal anterior. La explicación para el patrón de seromas en el muslo tiene más que ver con el movimiento del trocánter mayor con la deambulación.

La incidencia de seroma varía significativamente en la literatura, al igual que el enfoque para su prevención ${ }^{31}$. Se utilizan varios métodos para controlar la formación de seromas. Algunos cirujanos usan suturas tipo colchón para minimizar el espacio muerto y por lo tanto reducir el espacio disponible para la formación de seroma. Otros han utilizado selladores de tejidos durante el procedimiento ${ }^{17}$, pero todos concuerdan en el uso de drenajes. Los diferentes cirujanos manejan los drenajes de manera diferente.

Aly y cols. reportaron una tasa de $37,5 \%$ y describieron la eliminación de todos los drenajes en 2 semanas $^{5}$. Carwell y Horton, y Van Geertruyden y cols. describieron tasas de seromas del $14 \%$ y $6,6 \%$, respectivamente. En una serie de 40 casos, Pascal y Le Louarn no informaron seromas y eliminaron todos los drenajes 3 días después de la operación ${ }^{11}$.

En la técnica descripta anteriormente del levantamiento corporal, los drenajes se colocan a través del monte Venus en el pubis en un orden específico y en un lugar designado y lo habitual es comenzar a eliminar los drenajes 1 semana después de la cirugía. Por lo general, los dos drenajes que se abocan al abdomen se eliminan primero. Los drenajes se eliminan solo si drenan menos de $30 \mathrm{ml}$ en un período de 24 horas. La semana siguiente, los drenajes abocados a cada muslo se eliminan, y los drenajes de los glúteos se retiran aproximadamente a las 3 semanas.

Los drenajes glúteos también drenan los muslos. A las 5 semanas se retiran todos los drenajes independientemente de su débito. Saber dónde se coloca cada drenaje elimina la posibilidad de eliminar dos drenajes del mismo lado del cuerpo. La primera conducta frente al seroma es drenar la colección mediante aspiración con aguja. Si el paciente se presenta con algún signo o síntoma de infección o la calidad de los fluidos sugiere una infección, se envía el líquido para su análisis y se coloca un drenaje perforado de $10 \mathrm{~mm}$ en la cavidad del seroma por medio de la cicatriz del levantamiento corporal. Si el seroma mide más de $10 \mathrm{~cm}$ de diámetro y es clínicamente estéril, se le ofrece al paciente la posibilidad de que se coloque un drenaje en la cavidad. Otra opción es colocar un drenaje de Penrose en la cavidad del seroma por medio de la cicatriz. Para los pacientes que tienen que viajar largas distancias para visitas al consultorio, ésta puede representar la mejor op- 
ción. La formación de seroma se puede mantener en un nivel razonablemente bajo siguiendo un protocolo y con un cuidado de drenaje meticuloso ${ }^{31}$.

\section{Necrosis de la piel}

Los sitios más frecuentes de necrosis dependen de cuál haya sido el procedimiento quirúrgico empleado para el modelado del cuerpo. Así la región más afectada en la abdominoplastía en flor de lis es a nivel de la unión en $\mathrm{T}$ de las incisiones verticales y horizontales, pero en la abdominoplastIa en cinturón y en el procedimiento de levantamiento corporal son la región suprapúbica y, con menor frecuencia, las caderas y las hendiduras glúteas ${ }^{17}$. La necrosis suele ser el resultado de una mala circulación de los tejidos, que puede estar influenciada por variables como la tensión, el consumo de tabaco, las cicatrices, la liposucción y, en ciertos casos, la presión de los vendajes y las prendas de vestir. La necrosis a lo largo de la región suprapúbica del colgajo abdominal se puede explicar fácilmente por el origen aleatorio y periférico de su suministro de sangre después de la abdominoplastía.

La necrosis a lo largo de las caderas y las hendiduras glúteas, generalmente es marginal y puede tener más que ver con el efecto de la tensión en la perfusión tisular. En un esfuerzo por preservar el suministro de sangre a la porción hipogástrica del colgajo de la pared abdominal, limitamos tanto como sea posible la disección a nivel del epigastrio. Para la prevención de la necrosis marginal de la piel en las caderas consiste en aplicar solamente una mínima tensión en el muslo y el glúteo midiendo adecuadamente la cantidad de tejido a resecar.

Aunque se recomienda a los pacientes fumadores no fumar en el período perioperatorio, la mayoría solo disminuye su consumo y esto es un factor que puede influir negativamente en la oxigenación de los tejidos y por consecuencia aumenta las posibilidades de necrosis.

Las cicatrices abdominales superiores y en particular las subcostales, representan un factor de riesgo para la necrosis de la región inferior del abdomen, por lo tanto con éstos pacientes se debe tener especial cuidado en reducir al mínimo la disección en la región epigástrica. Si la región inferior del colgajo abdominal impresiona viable, se completa el procedimiento. Si existe duda sobre la viabilidad del colgajo abdominal durante el procedimiento, el área isquémica puede ser resecada de manera similar a la abdominoplastía en flor de lis.

El manejo de las necrosis limitadas se realiza con desbridamiento y curaciones locales. Las cicatrices que resultan en las áreas de necrosis pueden ser revisadas 1 año después de la cirugía.

La necrosis puede ser minimizada por: el uso juicioso de la disección y la liposucción de la región epigástrica, la marcación adecuada del tejido a resecar minimizando la tensión, y evitando el uso de las prendas de vestir que pueden afectar la circulación, en particular en el período posoperatorio temprano ${ }^{31}$.

\section{Infecciones}

Las infecciones son un problema infrecuente. La mayoría se presentan en pacientes que presentaron un seroma clínicamente no evidente o no diagnosticado y se infectaron. El tratamiento consiste en drenar la colección ya sea de manera abierta o colocando un drenaje de $10 \mathrm{~mm}$ en la cavidad. La patogénesis del seroma infectado no está claro. Una posibilidad es que se produce por los microorganismos de la piel que a través de los drenajes infectan el tejido desvitalizado, probablemente grasa. Por lo que mantener los drenajes por un período prolongado de tiempo resulta un riesgo.

Se sugiere mantener el esquema antibiótico hasta que se retiren los drenajes, aunque este régimen de antibióticos prolongado puede predisponer la infección por microorganismos más resistentes ${ }^{31}$.

Los pacientes que han sufrido un procedimiento de malabsorción, especialmente un bypass gástrico, no pueden absorber los antibióticos adecuados y por lo que puede requerir terapia intravenosa. Hemos manejado esta situación admitiendo a los pacientes, colocando una línea de catéter central insertada periféricamente, y luego continuando con los antibióticos intravenosos en su casa ${ }^{17}$.

\section{Hematoma / hemorragia}

El sangrado y la pérdida de sangre durante y después de los levantamientos corporales son un gran problema. $\mathrm{Mu}-$ chos aspectos de estos procesos predisponen a los pacientes a un riesgo de pérdida de sangre. Para tratar eficazmente la forma del contorno del cuerpo inferior de los pacientes con pérdida masiva de peso requiere una disección extensa de los tejidos, y con ello la necesidad de ligar o cauterizar muchos vasos sanguíneos. Una hemostasia meticulosa es fundamental en estos procedimientos.

Los pacientes con mayor peso, los hombres y aquellos con cambios más grandes en el IMC corren un mayor riesgo de pérdida significativa de sangre. Se debe evitar el uso rutinario de anticoagulantes en el período perioperatorio debido a la preocupación por el sangrado. Se recomienda a todos los pacientes que han sido sometidos a cirugía bariátrica que tomen suplementos de hierro cuando consideren la cirugía de contorno corporal $y$, en el caso de presentar anemia, se les deriba a un hematólogo. La incidencia de hematomas es baja 2,6\%.

Definimos un hematoma como una colección de sangre que requirió cirugía para su evacuación. Puede haber otros hematomas más pequeños que pasan desapercibidos o son evacuados por los propios drenajes ${ }^{31}$.

Trombosis venosa profunda y embolia pulmonar La trombosis venosa profunda y la embolia pulmonar representan el riesgo más grave para los pacientes que se someten a levantamientos corporales. Varios facto- 
res de riesgo reconocidos para la trombosis venosa profunda son fundamentales para estos procedimientos. En promedio, la población de pacientes tiene sobrepeso, y el levantamiento corporal es un procedimiento largo. Para complicar aún más las cosas, la ambulación temprana puede ser difícil, y el uso temprano y rutinario de anticoagulantes puede crear un riesgo significativo de hemorragia.

Para evitar la trombosis venosa profunda se debe proporcionar una anticoagulación mecánica continua hasta que el paciente sea ambulatorio. Los pacientes se mantienen en reposo en cama hasta el día posterior a la cirugía. Se les realiza un Doppler venoso de las extremidades inferiores el día del alta ${ }^{32}$. La incidencia de trombosis venosa profunda es del 2,4\%.

La embolia pulmonar es rara. El manejo de esta complicación potencialmente mortal en el paciente que se ha sometido a un levantamiento corporal presenta desafíos especiales. La heparinización del paciente postoperatorio temprano puede conducir a sangrado significativo. El momento y la dosificación de la heparina de- ben evaluarse cuidadosamente, por lo que podría ser necesario la colocación de un filtro de vena cava ${ }^{31}$.

\section{CONCLUSIÓN}

Luego de la pérdida masiva de peso en los pacientes que fueron sometidos a una cirugía bariátrica quedan estigmas muy visibles en su contorno corporal. Si bien estos están representados en varias regiones corporales, el abdomen es el primero en orden estadístico por el que los pacientes suelen recurrir a la consulta médica. Es fundamental un análisis exhaustivo prequirúrgico en estos pacientes para ofrecer los mejores resultados, y trabajar en un equipo multidisciplinario. En cuanto a la cirugía en sí, existen numerosas técnicas quirúrgicas para el tratamiento del abdomen de estos pacientes, por lo que de acuerdo a su clasificación deberíamos elegir la adecuada para optimizar resultados y prevenir complicaciones. El cirujano afronta un gran desafío en este tipo de cirugía, que representa una gran satisfacción para el paciente y su entorno como para el equipo quirúrgico.

\section{BIBLIOGRAFÍA}

1. Capella JF, Capella RF. Bariatric surgery in adolescence. Is this the best time to operate? Obes Surg 2003;13:826-32.

2. Capella JF, Capella RF. An assessment of vertical banded gastroplasty Roux-en-Y gastric bypass for the treatment of morbid obesity. Am J Surg 2002; 183:117-23.

3. Capella JF, Oliak DA, Nemerofsky RB. Body lift: an account of 200 consecutive cases in the massive weight loss patient. Plast Reconstr Surg 2006;117:414.

4. Aly AS, Cram AE, Heddens C. Truncal body contouring surgery in the massive weight loss patient. Clin Plast Surg 2004; 31:611-624.

5. Aly AS, Cram AE, Chao M, et al. Belt lipectomy for circumferential truncal excess: the University of low a experience. Plast Reconstr Surg 2003; 111(1):398-413.

6. http://www.oscardahir.com.ar/obesidadypacientespostbariaticos. php

7. https://rdu.unc.edu.ar/handle/11086/5021

8. Rubin JP, Nguyen V, Schwentker A. Perioperative management of the post-gastric-bypass patient presenting for body contour surgery. Clin Plast Surg 2004;31(4):601-10.

9. USDepartment of Agriculture. USDA National Nutrient Database for Standard Reference, release 17. Washington: USDA; 2004.

10. Van Way CW. Nutritional support in the injured patient. Surg Clin North Am 1991; 71:537-48.

11. Gleysteen JJ, Barboriak JJ. Improvement in heart disease risk factors after gastric bypass. Arch Surg 1983; 118:681-2.

12. Song $A Y$, Jean RD, Hurwitz DJ, et al. A classification of weight loss deformities: the Pittsburgh Rating Scale. Plast Reconstr Surg 2005; 116:1535-54.

13. Vastine VL, Morgan RF, Williams GS. Wound complications of abdominoplasty in obese patients. Ann Plast Surg 1999; 42:33-5.

14. Choban PS, Flancbaum L. The impact of obesity on surgical outcomes: a review. J Am Coll Surg 1997; 185:592-3.

15. Song $A Y$, Jean RD, Hurwitz DJ, et al. A classification of weight loss deformities: the Pittsburgh Rating Scale. Plast Reconstr Surg 2005; 116:1535-54.

16. O'Toole JP, Rubin JP, Matarasso A. Atlas of Aesthetic Surgery. Chapter 2: Evaluation of the Massive Weight Loss Patient Who Presents For Body Contouring Surgery. Elsevier. 2007.
17. Downy SE, Rubin JP, Matarasso A. Atlas of Aesthetic Surgery. Chapter 5: Aproach to the Abdomen After Weight Loss.

18. Jensen PL, Sanger JR, Matloub HS, et al. Use of a portable floor crane as an aid to resection of the massive panniculus. Ann Plast Surg 1990; 25:234-5.

19. Acarturk TO, Wachtman G, Heil B, et al. Panniculectomy as an adjuvant to bariatric surgery. Ann Plast Surg 2004; 53(4):360-6.

20. Matory WE, O'Sullivan J, Fudem G, et al. Abdominal surgery in patients with severe morbid obesity. Plast Reconstr Surg 1994; 94(7):976-87.

21. Richard EF. A mechanical aid for abdominal panniculectomy. $\mathrm{Br} J$ Plast Surg 1965; 18:336-7.

22. Hopkins MP, Shriner AM, Parker MG, et al. Panniculectomy at the time of gynecologic surgery in morbidly obese patients. Am J Obstet Gynecol 2000; 182:1502-1505. 18. Matarasso A, Wallach S. Abdominal contour surgery: treating all aesthetic units, including the mons pubis. Aesthetic Surg J 2001; 21(2):111-119.

23. Weihold S. Bauchdeck en plastic. Zentralb F Gynak 1909;38:1332.

24. Somalo M.: Dermolipectomia circular del tronco. Sem Méd 1940;1:1435.

25. Castañares S, Goethel J. Abdominal Lipectomy: A modification in technique. Plast Reconstr Surg 1967;40:378.

26. Regnault P. Abdominal dermolipectomies. Clin Plast Surg 1975; 2(3): 411.

27. Saldanha OR, et al. Lipoabdominoplasty without undermining. Aesth Surg J 2001; 21: 518. 6. Pontes R.: "Plástica Abdominal: Importância de sua associação à correção das hérnias incisionais". Rev Bras Cir 1966; 52: 85

28. Roxo, CDP. Abdominoplastia Multifuncional. Rev Soc Bras Cir Plast 2004;19(3):53.

29. Psillakis JM. Abdominoplasty: some ideas to improve results. Aesth Plast Surg 1978;2:205

30. Roxo C.P.D., Roxo, Almenares, M.C., Article: Abdominoplastia multifuncional: estudio retrospectivo de 5 años de experiencia con 108 pacientes Cirugía Plástica Ibero-Latinoamericana. Vol. 34:201218.2008.

31. Capella JF, Body Lift. Clinics in Plastic Surgery. Vol. 35:27-51, 2008.

32. Geerts WH, Heit JA, Clagett GP, et al. Prevention of venous thromboembolism. Chest 2001;119: 132S-75S. 